\title{
Small sensor probe for measuring plasma waves in space
}

\author{
Takahiro Zushi ${ }^{1}$, Hirotsugu Kojima ${ }^{2}$, Keisuke Onishi ${ }^{1}$, Mitsunori Ozaki ${ }^{3}$, Satoshi Yagitani ${ }^{3}$, Satoru Shimizu ${ }^{4}$ \\ and Hiroshi Yamakawa²
}

\begin{abstract}
Background: Since conventional one-point observations of plasma phenomena in space cannot distinguish between time and spatial variations, the missions on the basis of multiple-point observations have become the trend. We propose a new system for multiple-point observation referred to as the monitor system for space electromagnetic environments (MSEE).

Findings: The MSEE consists of small sensor probes that have a capability to measure electromagnetic waves and transfer received data to the central station through wireless communication. We developed the prototype model of the MSEE sensor probe. The sensor probe includes a plasma wave receiver, the microcontroller, the wireless communication module, and the battery in the 75-mm cubic housing. In addition, loop antennas, dipole antennas, and actuators that are used for expanding dipole antennas are attached on the housing. The whole mass of the sensor probe is $692 \mathrm{~g}$, and the total power consumption is $462 \mathrm{~mW}$. The sensor probe can work with both inner battery and external power supply. The maximum continuous operation time on battery power is more than $6 \mathrm{~h}$. We verified the total performance for electric field measurements by inputting signal to preamplifier. In this test, we found that analog components had enough characteristics to measure electric fields, and the A/D conversion and the wireless transmission worked correctly. In the whole performance for electric fields, the sensor probe has equivalent noise level of $-135 \mathrm{dBV} / \mathrm{m} / \sqrt{\mathrm{Hz}}$.

Conclusions: We succeed in developing the prototype model of the small sensor probe that had enough sensitivity for electric field to measure plasma waves and the ability to transfer observation data through wireless communication. The success in developing the small sensor probe for the measurement of plasma waves leads to the realization of the multiple-point observations using a lot of small probes scattered in space.
\end{abstract}

Keywords: Plasma wave receiver; ASIC; Multiple-point observation

\section{Findings Introduction}

Space is filled with dilute plasmas that are essentially collisionless. In collisionless plasmas, particles exchange their kinetic energies through plasma waves. This is addressed as wave-particle interactions. Wave-particle interactions control electromagnetic environments in space. Thus, since plasma waves reflect changes of electromagnetic environment in space, the observation of plasma waves is essential in monitoring the space electromagnetic environment, as well as for understanding physical processes taking place. Consequently, plasma waves have been observed

\footnotetext{
* Correspondence: kojima.hirotsugu.6m@kyoto-u.ac.jp

${ }^{2}$ Research Institute for Sustainable Humanosphere, Kyoto University, Gokasho, Uji 611-0011, Japan

Full list of author information is available at the end of the article
}

in the past scientific missions that target the investigation of space plasma phenomena.

Recently, missions to observe space plasma phenomena at multiple points have become the trend. The four Cluster II spacecraft were launched in 2000 by the ESA (Escoubet and Schmidt 2000), and NASA launched five THEMIS spacecraft in 2007 (Angelopoulos 2008). These multiple spacecraft missions were based on the idea that the multiple-point observation is essential in overcoming the disadvantage of one-point observation by a single satellite. Since one-point observation cannot distinguish between time and spatial variations of phenomena, the one-point observation of inhomogeneous phenomena has the defect. Space plasmas filling the terrestrial magnetosphere are strongly inhomogeneous in various spatial scales. The inhomogeneous spatial scales depend on plasma parameters such as Debye length and the

\section{Springer}

(c) 2015 Zushi et al. Open Access This article is distributed under the terms of the Creative Commons Attribution 4.0 International License (http://creativecommons.org/licenses/by/4.0/), which permits unrestricted use, distribution, and reproduction in any medium, provided you give appropriate credit to the original author(s) and the source, provide a link to the Creative Commons license, and indicate if changes were made. 
inertia length of electrons and ions. Relative distance between each spacecraft in multiple spacecraft missions should be decided while considering these characteristic parameters and the spatial scales of target phenomena.

The variation of plasma wave activities is sensitive to the variation of the plasma medium. Since plasma is a dispersive medium, the response of plasma waves appears as the excitation of wave modes along with their corresponding frequencies, wavenumbers, and polarizations. Identifying the mode of the generated plasma wave is a key in understanding the features of electromagnetic environments. Thus, multiple-point observations of plasma waves are crucial in understanding inhomogeneous plasma phenomena in space.

In addition to the inhomogeneity of natural phenomena in space plasmas, the interaction between plasmas and artificial structures also results in strong inhomogeneity. Gurnett et al. (1988) reported the existence of localized plasma wave turbulence and its inhomogeneity caused by the interaction between space plasmas and a space shuttle. In understanding the spatial distribution of such artificial plasma wave turbulence, multiple-point observations of plasma waves are also necessary.

In order to achieve multiple-point observation of plasma waves in space, Kojima et al. (2010) proposed a new system for monitoring the space electromagnetic environment. The proposed system is referred to as the monitor system for space electromagnetic environments (MSEE). The MSEE is a sensor system that consists of palm-sized sensor probes. Figure 1 shows a conceptual illustration of the proposed MSEE. Each sensor probe has the ability to measure plasma waves and transfer observation data to a central station, such as a satellite or a rocket (Kojima et al. 2010; Yagitani et al. 2011). Each sensor probe directly communicates with the central station. The central station gathers observation data and transfers the data to the ground. The sensor probes are randomly distributed throughout the target area to observe plasma waves from multiple points. Each sensor probe is completely dispensable and is not equipped with any thrusters or engines for controlling its attitude and orbit. The lifetime of the sensor probe ends when the probe penetrates the atmosphere. Since the MSEE sensor probe does not have the deorbit mechanism, its use is limited in the low altitude region around the Earth or the interplanetary region.

We succeeded in designing and developing the prototype model of the MSEE sensor probe. The sensor probe including electromagnetic antenna occupies a volume of $130 \mathrm{~mm}^{3}$ and has a mass of $692 \mathrm{~g}$. All of the electric circuits of the sensor probe are held in $75-\mathrm{mm}$ cubic housing. The sensor probe can measure electromagnetic waves with sensitivities that are sufficiently high to capture intense plasma wave such as electron cyclotron harmonic waves and transfer data wirelessly. The objectives of the present paper are to describe the detailed design of the MSEE sensor probe and to demonstrate its performance from the viewpoint of electric field measurements.

\section{Overview of the sensor probe}

A conceptual design of the sensor probe is presented in Kojima et al. (2010). Based on the conceptual design, we designed and manufactured the prototype model of the sensor probe.

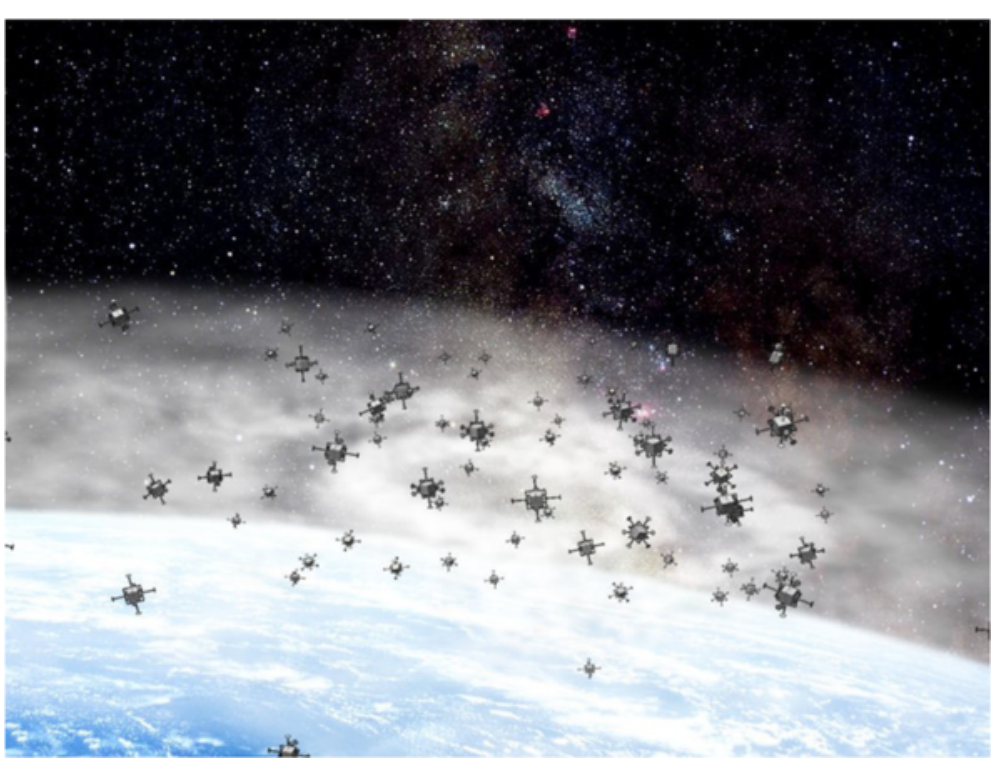

Fig. 1 Conceptual illustration of the proposed MSEE (Kojima et al. 2010) 
The following components are installed in a sensor probe:

(1)Electromagnetic sensors to pick up plasma waves

(2) Plasma wave receiver that filters and amplifies signals picked by sensors

(3)Digital processing unit to control the overall behavior of the sensor probe

(4) A wireless communication module to transfer data

(5)Battery

In addition, with the exception of the electromagnetic sensors, we package these components in a $75 \times 75 \times$ $75 \mathrm{~mm}$ housing in a sensor body.

The sensor probe loads three sets of electric sensors and tri-axial magnetic sensors (1). The "Electromagnetic sensors" section describes the details of the sensors. The plasma wave receiver (2) is composed of sensor preamplifiers and waveform receivers with six channels. We use the application-specific integrated circuit (ASIC) for the preamplifiers of magnetic field sensors and waveform receivers. While the design and performance of the ASIC waveform capture is described by Fukuhara et al.
(2011, 2012), the description of the ASIC preamplifier of the magnetic field sensor will appear in the independent paper (Ozaki et al. 2015). The conventional design is used for the preamplifiers of the electric field sensors, because of the necessity of very high impedance at the input. With regard to sample and hold circuits $(\mathrm{S} / \mathrm{H}$ circuits) and $\mathrm{A} / \mathrm{D}$ converters, we use the ones implemented within a dsPIC-series microcontroller. The dsPIC also functions as the CPU (3) above. The details of the analog circuits of waveform receiver with the preamplifier of the electric field sensors and dsPIC are written in the "Analog part of the plasma wave receiver" and "CPU as the digital processing system" section, respectively. For the wireless communication (4), we use the WH-MZ2 module developed by Oki Electric Industry Co., Ltd. Its details are written in the "Wireless communication module" section.

A block diagram of the overall sensor probe is shown in Fig. 2, and Table 1 lists the model numbers and power consumptions of the core components contained in the sensor probe. We do not describe the details of the loop antennas and their preamps. Three sets of preamplifiers of the loop antennas are implemented in one chip ASIC.

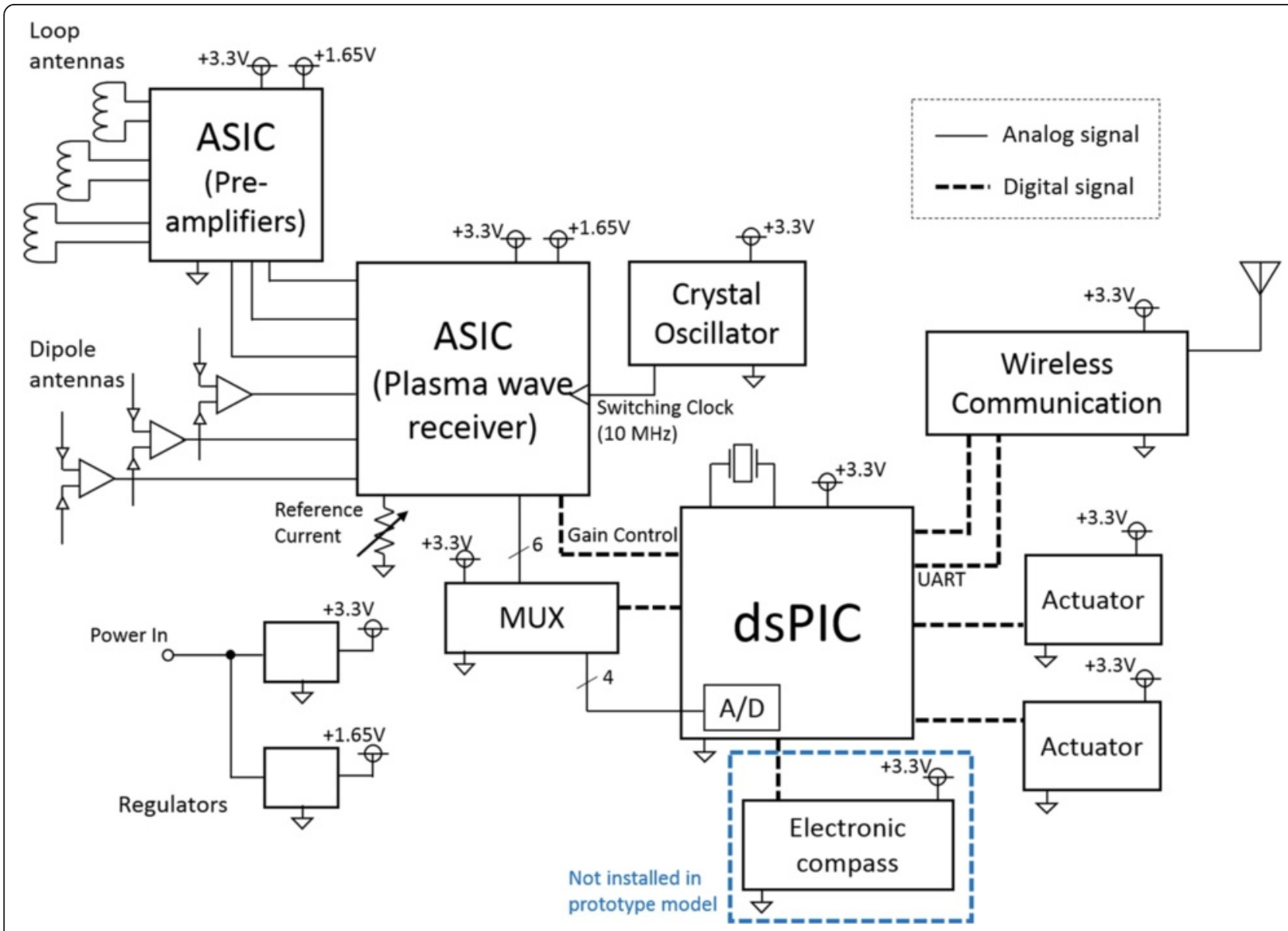

Fig. 2 Block diagram of the sensor probe 
Table 1 Model numbers of devices contained in the sensor probe

\begin{tabular}{llr}
\hline Device & Model number & Power consumption \\
\hline CPU & dsPIC33EP512GP504 & $<400 \mathrm{~mW}$ \\
Wireless communication & WH-MZ2 (Oki Electric Industry Co., Ltd.) & $<50 \mathrm{~mW}$ \\
ASIC (plasma wave receiver) & - & $<200 \mathrm{~mW}$ \\
\hline
\end{tabular}

The combination of the loop antennas and their ASIC preamplifiers are very unique. These will appear in the independent publication in the future (Ozaki et al. 2015). All of the electronic components operate at $3.3 \mathrm{~V}$, which is supplied by the regulator. A $1.65-\mathrm{V}$ reference voltage that is used in ASIC and preamplifiers is similarly supplied by another regulator. The main power source is selectable as a lithium-ion battery or an external power supply. Electromagnetic sensors are connected to the plasma wave receiver through preamplifiers. The output signals from the plasma wave receiver with six channels are connected to the dsPIC. However, the dsPIC has four channels of $\mathrm{S} / \mathrm{H}$ circuit. For this reason, a multiplexer is inserted between the plasma wave receiver and the dsPIC to select the measurement channels. The dsPIC controls the plasma wave receiver, the multiplexer, the actuators that are used for the expanding dipole antenna, and the wireless communication module. A universal asynchronous receiver/transmitter (UART) is used for communication between the dsPIC and the wireless communication module for transmitting measurement data.

The sensor probe generally performs two activities: plasma wave measurement and expansion of the dipole antenna. The details of plasma wave measurement and expansion of the dipole antenna are written in "CPU as the digital processing system" and "Electromagnetic sensors" section, respectively.

In order to attitude detection of a sensor probe by measuring geomagnetic field, we will implement the small electronic compass on the sensor probe. We plan to use the AMI306 (Aichi Micro Intelligent Corporation) which is 3-axis digital electronic compass. Magnetic field data measured by the electronic compass will be sent to the dsPIC through Inter-Integrated Circuit bus and finally send to central station together with plasma wave observation data. Note that the electronic sensor is mainly dedicated to the attitude detection not to the magnetic field fluctuation due to natural phenomena.

Using CMOS circuits in space, radiation tolerance needs to be considered. We verified that the ASIC waveform capture chip has enough radiation tolerance to radiation up to a total ionizing dose of several tens of krad. For single-event effect, radiation test for dsPIC and microcontroller of the wireless module is required for the future. According to the radiation test, local shielding is also planned.

\section{Electromagnetic sensors}

We adopt three sets of dipole antennas for the electric sensor and tri-axial loop antennas for the magnetic sensor. These sensors are dedicated to the measurement of full electromagnetic components of plasma waves. In order to minimize the size and the mass of the sensor probe in launching, the dipole antennas are made of carbon-fiber-reinforced plastic (CFRP) so as to be foldable. To fold and expand the dipole antennas, we plan to use actuators shaped like a padlock and a kind of strings. When the antenna is folded, the antenna is wound around the frame attached to the housing of the sensor probe and is held by a string (see Fig. 3). Both ends of the string are looped and held by the actuator. When the actuator is activated, it becomes unlocked and the string becomes free. Then, the unfastened antenna is deployed automatically by its self-tension. The timing of deploying antennas is controlled by external signal sent

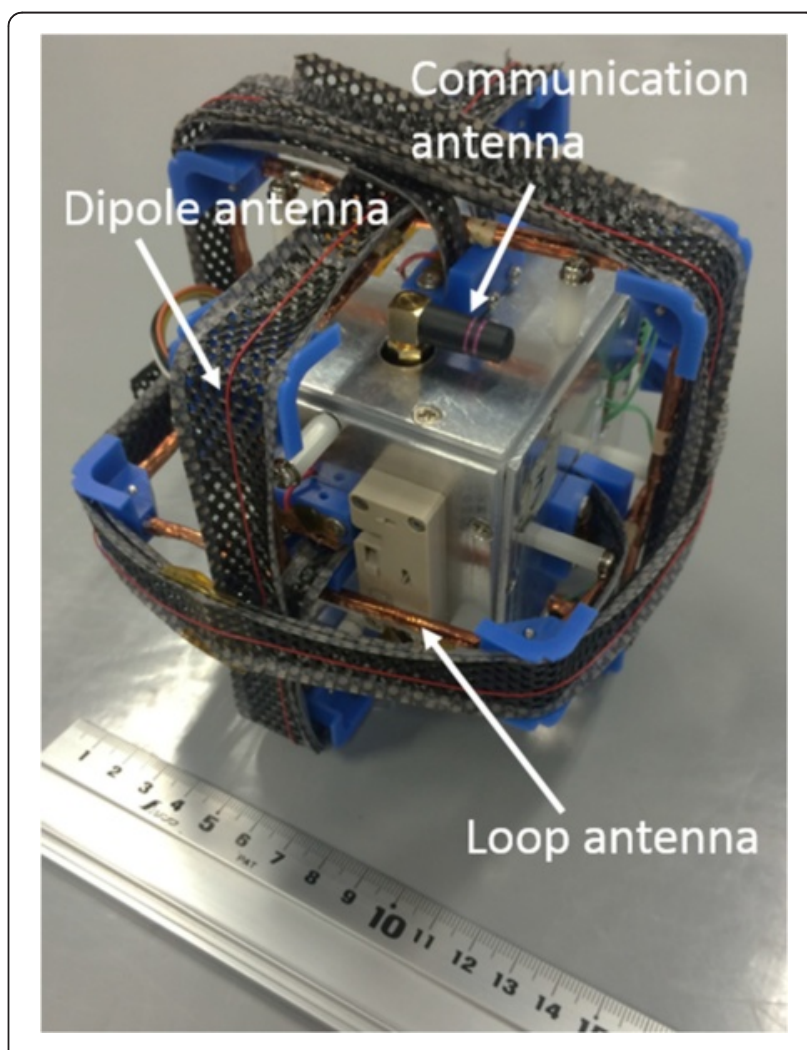

Fig. 3 Photograph of the sensor probe with folded antennas 
from central station to the sensor probe. When the sensor probe receives the signal wirelessly, the dsPIC activates the actuator to expand antenna.

Figure 3 shows the electromagnetic sensors installed outside the sensor probe. The small communication antenna for $2.45 \mathrm{GHz}$ is also seen in Fig. 3. It is used for wireless communication, and its details are written in "Wireless communication module" section.

\section{Analog part of the plasma wave receiver}

The role of the analog circuit in plasma wave receiver is to filter and amplify the target signals picked up by sensors to the ones with the enough intensity to apply analog-to-digital conversion. The ASIC realizes a chipsize plasma wave receiver with the size of a few millimeters $\times$ a few millimeters. The ASIC we developed includes the analog circuits such as filters and amplifiers required for a plasma wave receiver in one chip. The detailed specification and the performance of the plasma wave chip are described by Fukuhara et al. (2012). Although we improved the chip design for the use in the prototype of the sensor probe, basic design was the same with that by Fukuhara et al. (2011).

Figure 4 shows a block diagram of the ASIC of the plasma wave receiver. The design of the ASIC is based on that developed by Fukuhara et al. (2011). In the version used in the prototype of the sensor probe, we add the adjust circuit of the output offset and improve the stability of the chip. The ASIC includes six channels of circuits that are composed primarily of an amplifier and three filters. The role of the first low-pass filter (LPF) is band limiting. Since the LPF is in front of the amplifier, the LPF has a low-noise characteristic. The second component of the circuit is a main amplifier that three gain steps: 0,20 , and $40 \mathrm{~dB}$. The gain for the electric and magnetic fields can be changed independently by external control signals. The next filter is an anti-aliasing filter. The anti-aliasing filter is a discrete-time filter and so requires a clock signal and generates switching noise. For this reason, a noise-eliminating filter is placed behind the anti-aliasing filter. The filter type of the antialiasing filter is a sixth-order Chebyshev.

Two types of filter are adopted in the ASIC: a Gm-C filter and a switched capacitor (SC) filter. Since Gm-C filters have relatively low-noise characteristics and do not require an external signal, a $\mathrm{Gm}-\mathrm{C}$ filter is used to realize a band-limiting LPF and a noise-eliminating LPF. On the other hand, the SC filter requires a clock signal but can realize high accuracy and steep-eliminating characteristics beyond the cutoff frequency. For this reason, an SC filter is used as an anti-aliasing filter that requires a cutoff frequency of $100 \mathrm{kHz}$ with high stability. The cutoff frequency of the SC filter can be controlled by the change of the external clock.

The typical frequency response of the ASIC is shown in Fig. 5. Within the pass band, gains of 0.2, 18.2, and $38.3 \mathrm{~dB}$ are observed for each gain setting. The reduction characteristics at frequencies over $100 \mathrm{kHz}$ are attributed to the anti-aliasing filter. The upper cutoff frequency is changeable in relation to the sampling frequency by changing its clock frequency.

Figure 6 shows a schematic of the preamplifier of the electric field sensors. The preamplifier is composed of discrete electronic parts. The preamplifier of the electric field sensor is essential in picking up electric field component of plasma waves. The impedance of electric field sensor in plasmas strongly depends on plasma parameters such as densities and temperatures. In order to pick up the electric component of plasma waves effectively, the input impedance of the preamplifier should be higher than that of the electric field sensor. In the present design, the input impedance is $220 \mathrm{Mohm}$. Furthermore, present preamplifier is designed as a twostage amplifier. The first stage is a differential amplifier composed of two field effect transistors (FET) Tr1, Tr2 and an operational amplifier (op-amp) OP-AMP1. To realize high sensitivity, signals picked up by antennas are fed to the FET input differential amplifier with the gain of about $20 \mathrm{~dB}$. The final stage is inverting amplifier composed by OP-AMP2. The gain of the inverting

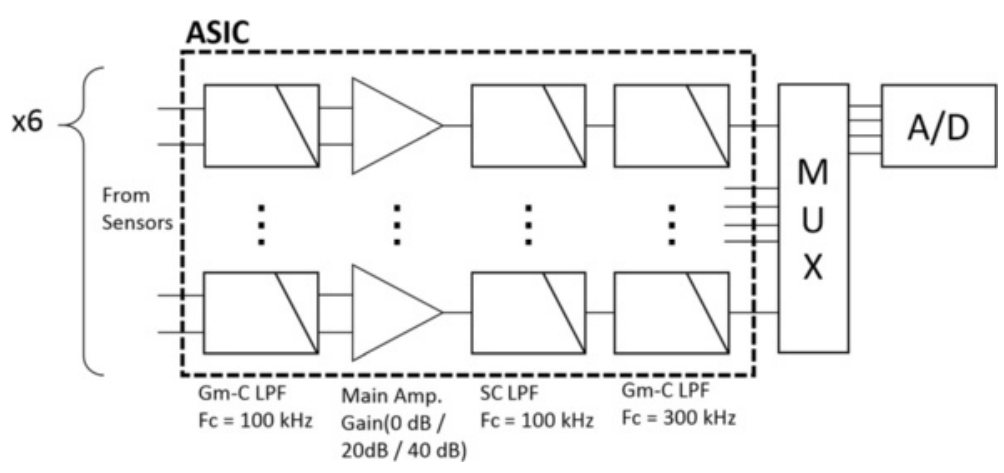

Fig. 4 Block diagram of the ASIC for plasma wave receiver 


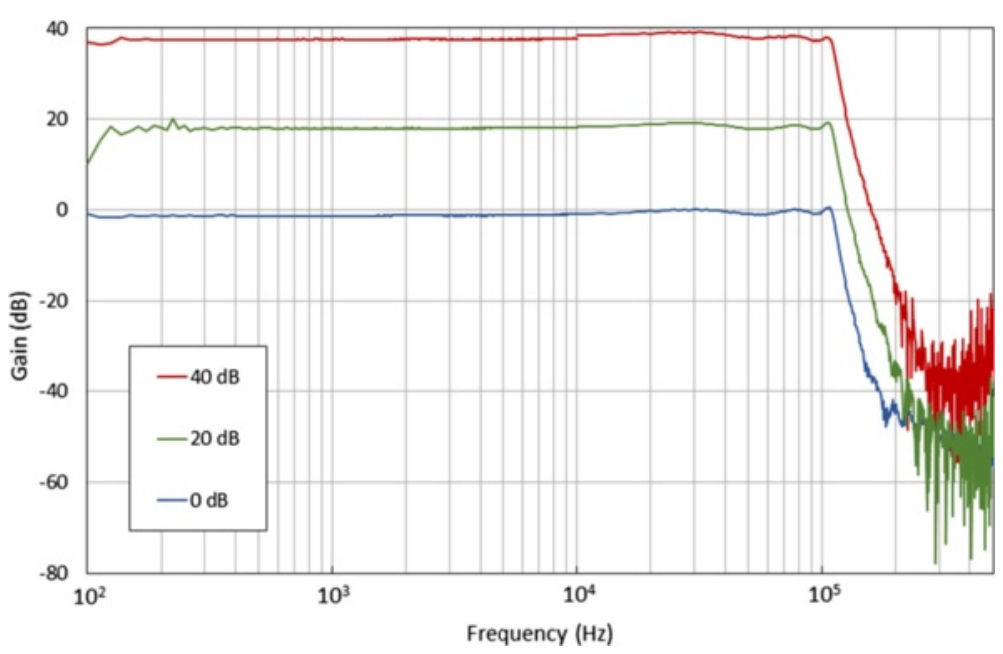

Fig. 5 Typical frequency response of the ASIC

amplifier is given as R11/R10. The gain of the OPAMP2 should be changed depending on the mission target. In the present design shown in Fig. 6, the gain of the OP-AMP2 is $20 \mathrm{~dB}$. The whole frequency response from the input of the preamplifier through the output of the ASIC is shown in Fig. 7. In this measurement, the gain of the ASIC is set to $0 \mathrm{~dB}$. Thus, the total gain from the input of the preamplifier of electric field components to the output of the ASIC is $36 \mathrm{~dB}$. The reduction of the gain until the frequency of $1 \mathrm{kHz}$ is due to that capacitors $\mathrm{C} 4, \mathrm{C} 5$ and resistors connected to them act as highpass filter. By choosing the capacitors $\mathrm{C} 4, \mathrm{C} 5$ and the resisters R5, R7, we can adjust the lower cutoff frequency of the preamplifier. In general, plasma waves in

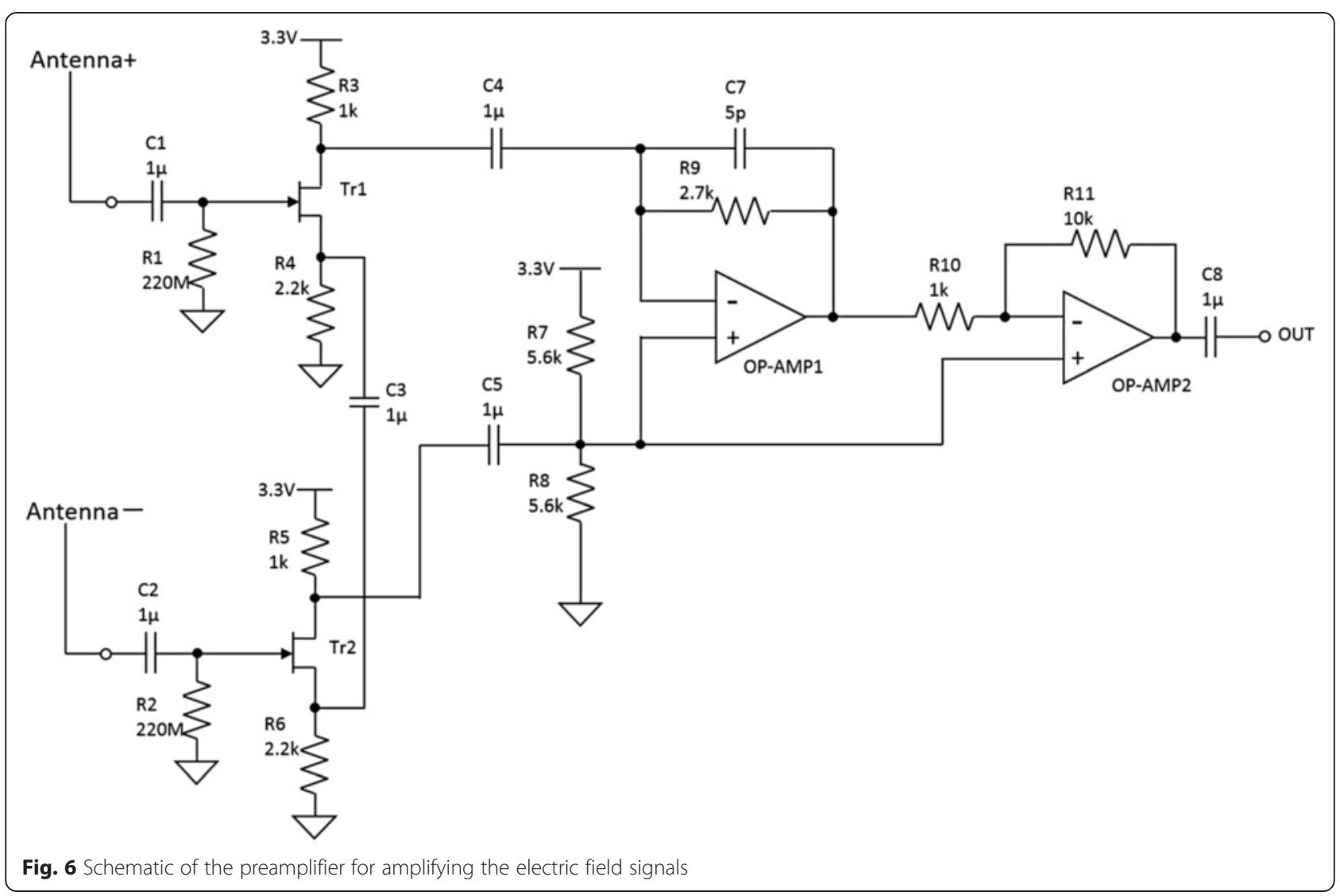




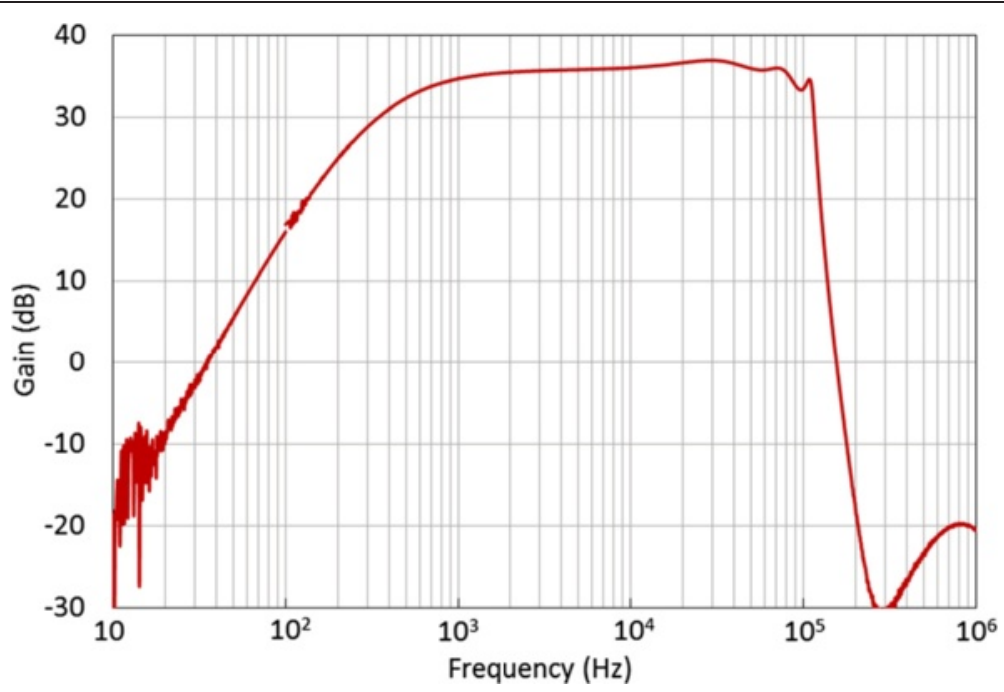

Fig. 7 Frequency response of the preamplifier and the ASIC

lower frequency range are more intense than the ones in higher frequency range. The decrease of the gain as shown in Fig. 7 is reasonable in the design of preamplifiers.

As stated in the previous section, the preamplifiers of the loop antennas are developed as one chip system using ASIC. The details will soon appear in the independent paper (Ozaki et al. 2015).

\section{CPU as the digital processing system}

In order to keep the phase relation between different measurement channels, the simultaneous $\mathrm{S} / \mathrm{H}$ circuits in front of $\mathrm{A} / \mathrm{D}$ converters are essential. Considering the number of $\mathrm{S} / \mathrm{H}$ circuits and the size of the inner memory, dsPIC33EP512GP504 (Microchip Technology Inc.) is used in the sensor probe, which includes four $\mathrm{S} / \mathrm{H}$ circuits, a 10-bit A/D converter, and $16 \mathrm{kB}$ of RAM.

The primary functions of the dsPIC are carrying out A/ D conversion and transferring data to the wireless communication module. In order to realize high-frequency sampling and data transmission, $\mathrm{A} / \mathrm{D}$ conversion needs to be intermittent. As such, the dsPIC carries out 10-bit A/D conversion at $198 \mathrm{kSample} / \mathrm{s}$ and stores the data in RAM. When the dsPIC stores 2048 data points for four channel, the entirety of stored data is transferred to wireless communication module all at once. The max number of store data depends on RAM size. The dsPIC cannot perform simultaneous sampling of all electromagnetic components because the dsPIC has only four channels of the S/H circuit. Thus, three channels of the electric field and the $x$ axis of the magnetic field or three channels of the magnetic field and the $x$-axis of the electric field are measured and data transfer is performed. The measured components can be changed during the process intervals.
Similarly, the gain of the ASIC can be changed during the process intervals.

\section{Wireless communication module}

For the wireless communication between a sensor probe and a central station, the wireless communication module WH-MZ2 (Oki Electric Industry Co., Ltd.) and the dedicated communication antenna with the length of $5 \mathrm{~cm}$ for $2.45 \mathrm{GHz}$ are used in the sensor probe. WHMZ2 is a system-on-chip device for $2.45 \mathrm{GHz}$ wireless communication and includes an ARM Cortex-M0 32-bit $\mathrm{CPU}$, a $2.45 \mathrm{GHz}$ radio module, $\mathrm{I} / \mathrm{O}$ modules, and $16 \mathrm{kB}$ of RAM.

The primary functions of the wireless communication module are to transfer data received from the dsPIC and to trigger the dsPIC when the signal for antenna expansion is received from a central station. In transferring data, the wireless communication module waits for the data from the dsPIC through the UART and store the data until the number of data reaches 2048. Then, the stored data are transmitted at $2.45 \mathrm{GHz}$. The bit-rates of UART communication and radio communication are $115,200 \mathrm{bps}$ and $250 \mathrm{kbps}$, respectively.

Table 2 shows the specification of the wireless communication module and the communication antenna used in the prototype of the sensor probe. The transmissible distance depends on the performance of the receiving

Table 2 Specification of the wireless communication module and the monopole antenna

\begin{tabular}{ll}
\hline Gain of the communication antenna & $1.6 \mathrm{dBi}$ \\
Power loss in the antenna & $<0.5 \mathrm{~dB}$ at $2.45 \mathrm{GHz}$ \\
Output power of the transmitter & $0 \mathrm{dBm}$ \\
Sensitivity of the receiver & $-96 \mathrm{dBm}$ \\
\hline
\end{tabular}


antenna. When we use the same antenna at the receiver, the sensor probe can be expected to communicate over $500 \mathrm{~m}$ in free space.

\section{Prototype of the sensor probe with electromagnetic sensors}

Figure 8 shows an external view of the prototype of the sensor probe under the deployment of the electric field sensors with the length of $1.6 \mathrm{~m}$ tip-to-tip. Three sets of dipole antennas, tri-axial loop antennas, a wireless communication antenna, and actuators, are attached to the housing, and the other components are installed inside the housing. The housing of the sensor probe is cubic and has sides of $7.5 \mathrm{~cm}$, and the loop antennas are square and have sides of $11 \mathrm{~cm}$. The total mass of the sensor probe is $692 \mathrm{~g}$.

The sensor probe can be powered by either the built-in battery or an external $7 \mathrm{~V}$ power supply. The built-in battery is the Li-ion type battery with the capacity of $1200 \mathrm{mAh}$. When the sensor probe is powered by the battery, it can measure electromagnetic waves and transfer the observed data for over $6 \mathrm{~h}$. When powered by an external power supply, the current is approximately $140 \mathrm{~mA}$.

\section{Total performance of the measurement electric field component}

Since numerous unwanted radio waves are present near ground level, it is difficult to verify the performance of the sensor probe by measuring existing radio waves such as the standard time and frequency signal. Thus, we conduct performance tests using an external signal source. Figure 9 shows the test configuration of the total performance test. The external signal source stimulates an input of the preamplifier of electric field channel without the electric field sensors. The output signal of the preamplifier is filtered and amplified by the ASIC of the plasma wave receiver and digitized by the A/Ds of the dsPIC. The digitized waveforms are transmitted through the wireless communication module to another module to which the output is connected with the PC through the serial interface. The received data are stored on the PC and we can reproduce the waveform of the external signal source. In a real flight in space, this

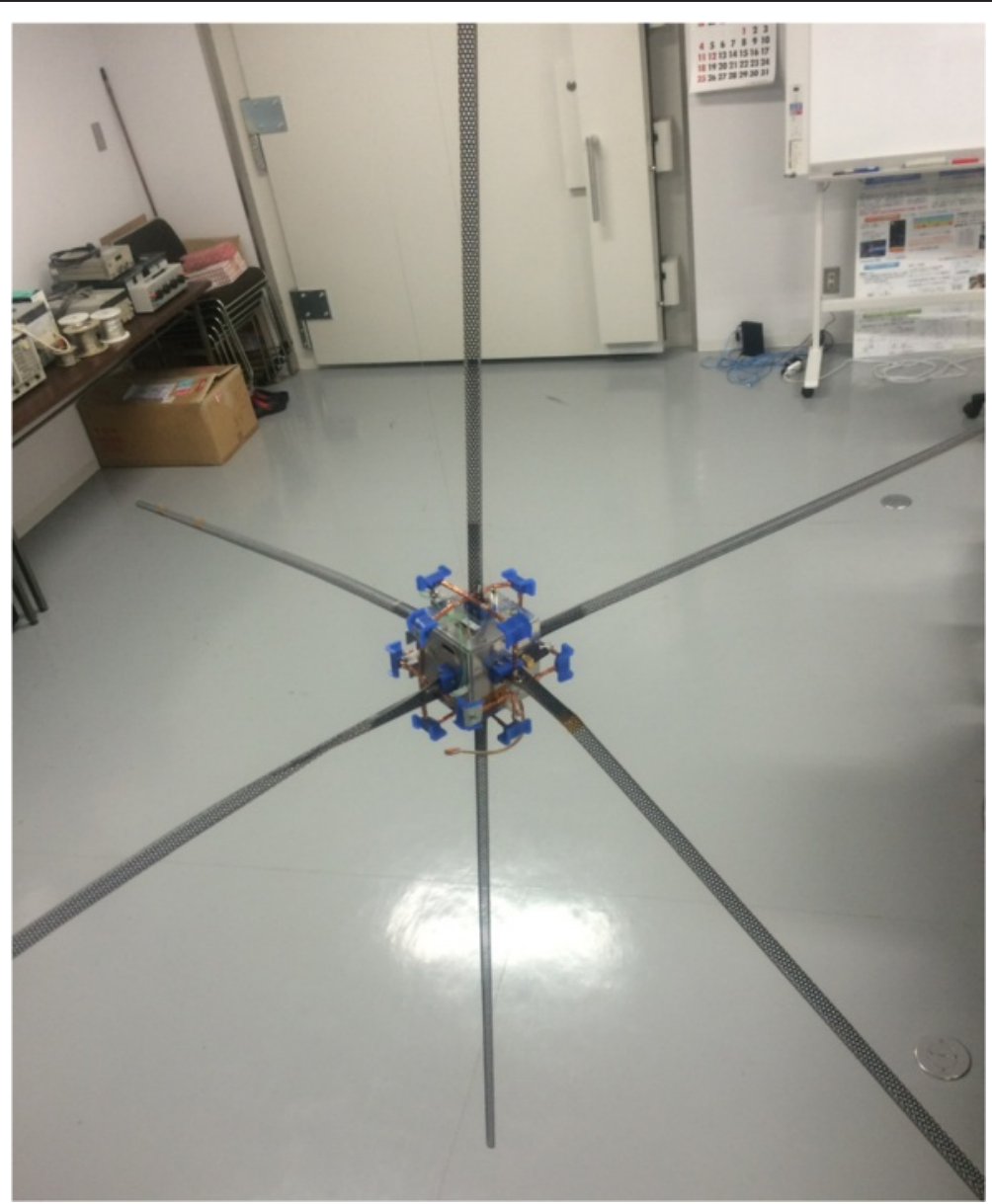

Fig. 8 Prototype model of the sensor probe with electromagnetic sensors 


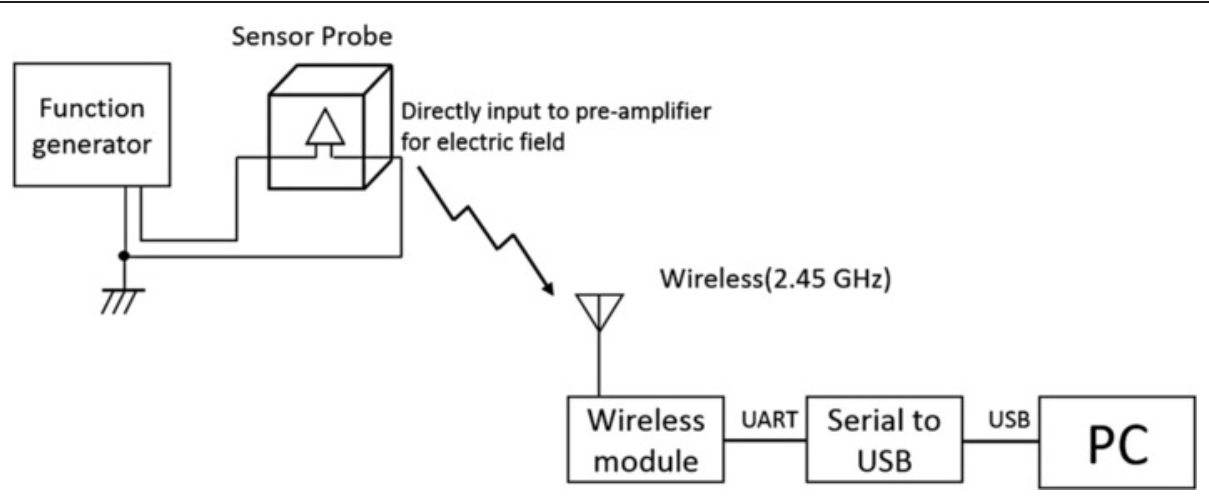

Fig. 9 Schematic diagram of electric field measurement

receiving wireless module and the $\mathrm{PC}$ are equivalent to a spacecraft or rocket as a central station.

Figure 10 shows one example of the reproduced waveform using the transmitted data from the MSEE sensor probe. The frequency of the external signal source is $9 \mathrm{kHz}$, and its amplitude is $-43 \mathrm{dBV}$. The gain of the ASIC of the plasma wave receiver is set to $0 \mathrm{~dB}$. In Fig. 10, the vertical axis shows the output voltage of the ASIC (input voltage at the A/D of the dsPIC), which is calculated from the received digital value. Figure 11 shows the amplitude spectrum of the received data shown in Fig. 10 as the result of the fast Fourier transform (FFT). This figure shows that the amplitude at a frequency of $9 \mathrm{kHz}$ is $-7 \mathrm{dBV}$. Based on Fig. 7, the total gain of the system is $35.9 \mathrm{~dB}$ at a frequency of $10 \mathrm{kHz}$. Using this value, it is expected that the amplitude of the output voltage is $-7.1 \mathrm{dBV}$. Thus, the electric field measurement is correctly carried out. Figure 12 shows the frequency response calculated by received data. In Fig. 12, we can see that there is little difference between the gain calculated and directly measured over the whole measuring frequency band. The difference between the received data and the expectation is probably due to the noise included in the generated signal. The DC component of the data calculated by FFT is $1.62 \mathrm{~V}$, which is reasonable for the ASIC output. The difference from $1.65 \mathrm{~V}$, which is the signal common voltage, is due to the mismatched properties of the ASIC.

Figure 13 shows the equivalent input noise level that is average of eight data calculated by received data with the input terminal of the preamplifier short and $20 \mathrm{~dB}$ gain setting in ASIC. In this calculation, we assume the insertion loss of $8 \mathrm{~dB}$ at the input of the preamplifier and the effective length of the electric field sensor of $0.8 \mathrm{~m}$. The noise level around a few tens of $\mathrm{kHz}$ is almost $-135 \mathrm{dBV} / \mathrm{m} / \sqrt{\mathrm{Hz}}$. This sensitivity of the electric field is enough to observe the intense plasma waves in space. In Fig. 13, there were two peaks in 58 and $74 \mathrm{kHz}$. The cause of these peaks is radiate noise from the external source. Consequently, no noise interferes with the electric field measurement exists inside the MSEE sensor probe. Generally, the preamplifier and the

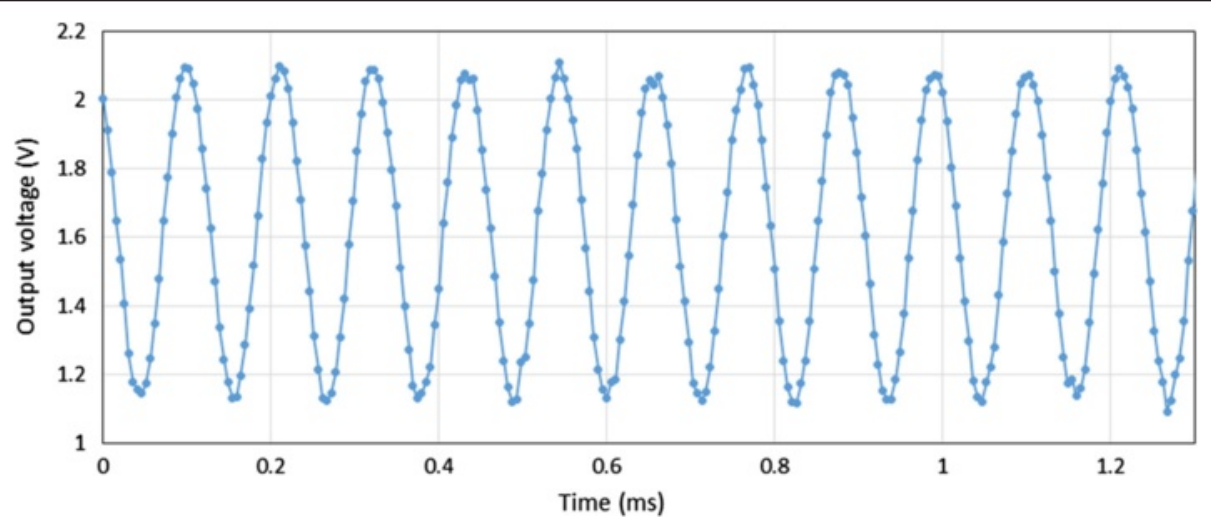

Fig. 10 Received data of the electric field measurement 


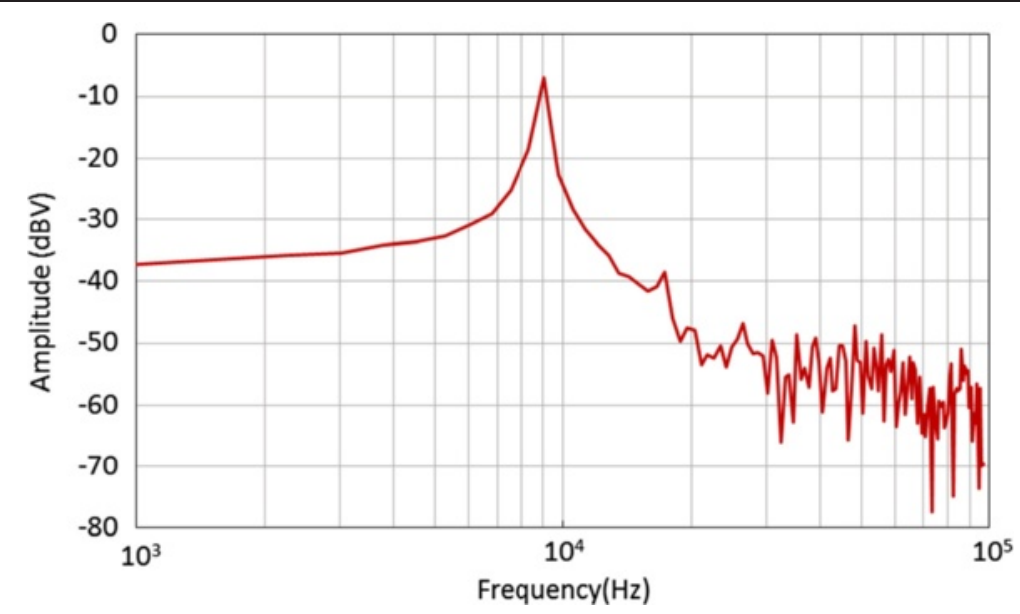

Fig. 11 Amplitude spectrum of the received data in electric field measurement

ASIC have characteristics sufficient for measuring electric fields, and the $\mathrm{A} / \mathrm{D}$ conversion and the wireless transmission work correctly.

\section{Conclusions}

We have developed the prototype of the small sensor probe that has a capability to measure electromagnetic waves and transmit observation data by the wireless. The prototype of the sensor probe contains the ASIC for plasma wave receiver to miniaturize as well as the sensor preamplifiers, wireless communication module, and other necessary components. The size of the housing is $75 \mathrm{~mm}$ cubic. The electromagnetic sensors are installed outside the housing. The tri-axial loop antennas and three sets of the self-deployable dipole antennas are used for the sensors. The total size of the sensor probe is $110 \mathrm{~mm}$ cubic and the mass is $692 \mathrm{~g}$ before the deployment of the electric field sensor. Once the electric field sensor is deployed, its length is $1.6 \mathrm{~m}$ tip-to-tip. The circuit block and its design were described. In particular, the success in developing the chip of the plasma wave receiver is crucial to realize the small housing of the sensor probe.

We showed the total performance and the noise level using the telemetered data from the sensor probe in the view point of electric field measurement. The result demonstrated that the miniaturization of analog part enable to realize small instruments for plasma waves. Consequently, sensor probes are expected to be a new way to measure plasma waves at multiple points in space.

In the present paper, we showed the design and performance of the prototype of the sensor probe, which is dedicated to measure plasma waves in the view point of the measurement of electric field components. Characteristics of the electromagnetic sensors are not discussed

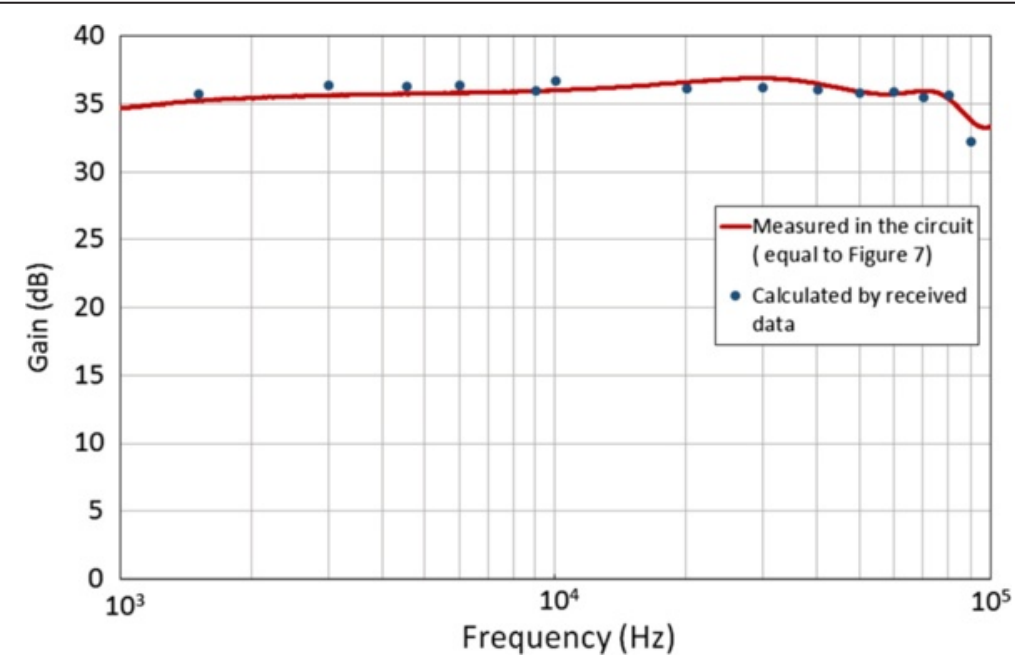

Fig. 12 Frequency response calculated by received data 


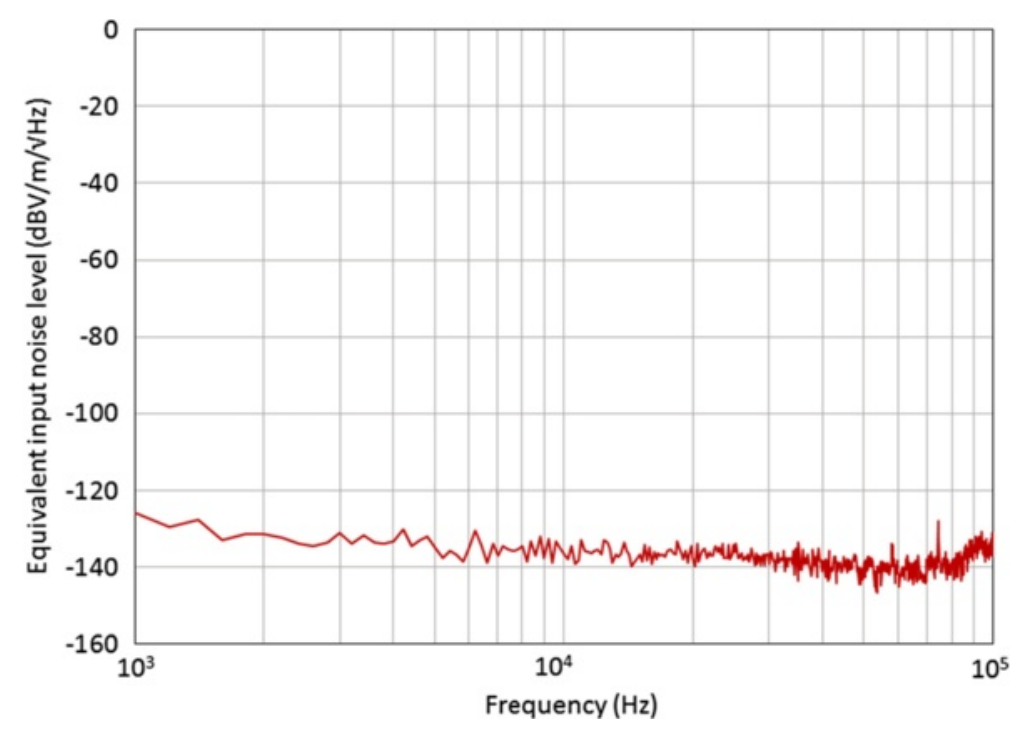

Fig. 13 Amplitude spectrum of measurement data with the input terminal short

in this paper. The performances of the first version of the electromagnetic sensors are introduced in Kojima et al. (2010) and Yagitani et al. (2011). While the design of the electric field preamplifier is basically the same with that of Kojima et al. (2010), the preamplifiers of the loop antennas in the present prototype are newly designed and implemented in the ASIC chip. The details of the loop antennas and their ASIC preamplifiers will appear in the independent paper (Ozaki et al. 2015).

The current prototype model does not have the locator system of the sensor probe. Basically, we plan to use a small GPS receiver. When we need to know more precise position in each probe than the position decided by GPS or we cannot use the GPS system in space such as the interplanetary region, another locator method should be considered. The locator method of small sensors without using GPS is a significant topic in the ground-based sensor network system as well as that in space. Many attempts have been made by using small communication system installed inside each small sensor (e.g., Takizawa et al. 2012). Some of them can be applied to the MSEE sensor probe. We will consider and implement the localization algorithm to our sensor probe system as required.

The sensor probes which we introduced in the present paper are distributed in a target area in space. They allow us to collect waveform data observed simultaneously at multiple points. One of the important target areas in the terrestrial magnetosphere which the MSEE system is applied to is the cusp region. The ion outflow and associated perpendicular heating in the cusp region are considered as the result of wave-particle interactions. A lot of sounding rockets and satellite observations have been conducted to investigate the mechanism of the perpendicular heating (e.g., Kintner et al. 1996). However, the mechanism is still unclear. One of the reasons is the difficulty in distinguishing time variation and spatial variation in the source region. Thus, we need more sophisticated multiple-point observation in the cusp region and the MSEE system has the potential in making a breakthrough in the study of the transverse ion acceleration. The MSEE system will be proposed in future cusp missions.

\section{Availability and requirements}

Project name: none

Project home page: none

Operating system(s): none

Programming language: none

Other requirements: none

License: none

Any restrictions to use by non-academics: none

\section{Competing interests}

The authors declare that they have no competing interests.

\section{Authors' contributions}

TZ developed ASIC for plasma wave receiver and wrote the manuscript under the guidance by HK and HY. HK conceived the idea of the MSEE and jointly wrote the manuscript. TZ, HK, and KO performed the general design of the sensor probe. MO and SY developed ASIC for preamp and loop antennas. SS developed the small wireless communication module. AW and HI developed the CFRP electric field sensor. All authors read and approved the final manuscript.

\section{Acknowledgements}

We are grateful to Mr. A. Watanabe and Mr. H. Ito of Sakase Adotech Corporation for fabrication of the CFRP electric field sensor. The present study was partially supported by the VLSI Design and Education Center (VDEC) of the University of Tokyo in collaboration with Cadence Design Systems, Inc. and SiliConsortium Ltd. The fabrication of the printed circuited board and the sensor probe housing were supported by Meiwa System Corporation. The performance test of the MSEE sensor probe was conducted 
in the PEMSEE system of Research Institute for Sustainable Humanosphere, Kyoto University. Additional support was provided by a grant-in-aid for Japan Society for the Promotion of Science (JSPS) Fellows, grants-in-aid for Scientific Research A (23244097).

\section{Author details}

'Department of Electrical Engineering, Graduate School of Engineering, Kyoto University, Kyotodaigakukatsura, Kyoto 615-8530, Japan. ${ }^{2}$ Research Institute for Sustainable Humanosphere, Kyoto University, Gokasho, Uji 611-0011, Japan. ${ }^{3}$ Graduate School of Natural Science and Technology, Kanazawa University, Kakumamachi, Kanazawa 920-1192, Japan. ${ }^{4}$ Oki Electric Industry Co., Ltd., Toranomon, Minato-ku, Tokyo 105-0001, Japan.

Received: 4 February 2015 Accepted: 1 August 2015

\section{Published online: 14 August 2015}

\section{References}

Angelopoulos V (2008) The THEMIS mission. Space Sci Rev 141:5-34. doi:10.1007/ s11214-008-9336-1

Escoubet CP, Schmidt R (2000) Cluster II: plasma measurements in three dimensions. Adv Space Res 25:1305-1314. doi:10.1016/50273-1177(99)00639-0

Fukuhara H, Kojima H, Okada S, Ikeda H, Yamakawa H (2011) Toward a waveform receiver on a chip dedicated to plasma wave instrument onboard scientific spacecraft. Aerospace Conference IEEE 2011:1-9. doi:10.1109/ AERO.2011.5747462

Fukuhara H, Kojima H, Ishii H, Okada S, Yamakawa H (2012) Tiny waveform receiver with a dedicated system chip for observing plasma waves in space. Meas Sci Technol 23:105903. doi:10.1088/0957-0233/23/10/105903

Gurnett DA, Kurth WS, Steinberg JT, Shawhan SD (1988) Plasma wave turbulence around the shuttle: results from the spacelab-2 flight. Geophys Res Lett 15:760-763. doi:10.1029/GL015i008p00760

Kintner PM, Bonnell J, Arnoldy R, Lynch K, Pollock C, Moore T (1996) SCIFERTransverse ion acceleration and plasma waves. Geophys Res Lett 23:1873-1876

Kojima H, Fukuhara H, Mizuochi Y, Yagitani S, Ikeda H, Miyake Y, Usui H, Iwai H, Takizawa Y, Ueda Y, Yamakawa H (2010) Miniaturization of plasma wave receivers onboard scientific satellites and its application to the sensor network system for monitoring the electromagnetic environments in space. Adv Geosci 21:461-481

Ozaki M, Yagitani S, Kojima H, Takahashi K, Koji H, Zushi T, Tokunaga Y (2015) Development of ASIC preamplifier for electromagnetic sensor probes toward monitoring space electromagnetic environments, to be submitted to Earth, Planets and Space

Takizawa Y, Ohno S, Adachi N (2012) Self-organizing localization for wireless sensor networks based on RSSI. Sens Lett 10:1-10

Yagitani S, Ozaki M, Kojima H (2011) A compact loop antenna system for monitoring local electromagnetic environments in geospace. IEICE Trans Commun E94-B:1744-1747

\section{Submit your manuscript to a SpringerOpen ${ }^{\circ}$ journal and benefit from:}

- Convenient online submission

- Rigorous peer review

- Immediate publication on acceptance

- Open access: articles freely available online

- High visibility within the field

- Retaining the copyright to your article 